Relato de Experiência

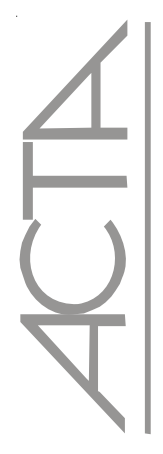

\title{
Implantação de sistema informatizado para planejamento, gerenciamento e otimização das escalas de enfermagem*
}

\author{
Implementation of a computerized system to plan, manage, and optimize nursing staff schedule \\ Implantación de un sistema informatizado para la planificación, administración y optimización en \\ la elaboración de los turnos de enfermería
}

\author{
Ana Cristina Rossetti ${ }^{1}$, Lucio Mauro Carqui ${ }^{2}$
}

\begin{abstract}
RESUMO
Objetivo: Relatar o desenvolvimento e implantação de um sistema informatizado para auxiliar no planejamento, gerenciamento e otimização das escalas de enfermagem nas unidades de internação do Hospital Israelita Albert Einstein. Métodos: Foi desenvolvido o Sistema de

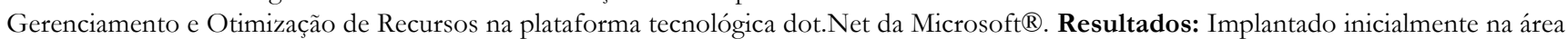
de enfermagem com visualização on-line das escalas das unidades e respectivas demandas de pacientes. Facilita e permite a elaboração e o gerenciamento das escalas mensais de trabalho, reduzindo o tempo utilizado na confecção e garantindo segurança na informação e agilidade na obtenção de dados. Conclusão: Após a implantação é possível observar ganhos e oportunidades de melhoria tanto no sistema como no processo de planejamento e gerenciamento das escalas de enfermagem.

Descritores: Recursos humanos de enfermagem no Hospital; Sistemas de informação; Admissão e escalonamento de pessoal; Sistemas de informação para admissão e escalonamento de pessoal
\end{abstract}

\begin{abstract}
Objective: To report the development and implementation of a computerized system that supports the planning, management, and optimization of nursing staff schedule for inpatient units of the Israelite Albert Einstein hospital. Methods: The technological platform dot.Net by Microsoft ${ }^{\circledR}$ was used to develop the Resource Management and Optimization System (SGOR). The SGOR was initially implemented in the nursing units to allow online visualization of nursing schedule per unit and shift according to respective patients' demand. Results: The SGOR was found to facilitate the management of monthly nursing schedules, to decrease design time, to provide safety and accurate information, and to allow quick data retrieval. Conclusion: The implementation of the SGOR is a useful system for the management of nursing staff schedule. It allows determining improvements in the schedule of nursing staff as well as determining the need for improvements in both the system itself and the planning and management of nursing staffing demand.

Keywords: Nursing staff, hospital; Information systems; Personnel staffing and scheduling; Personnel staffing and scheduling information systems
\end{abstract}

\section{RESUMEN}

Objetivo: Relatar el desarrollo e implantación de un sistema informatizado para auxiliar en la planificación, administración y optimización en la elaboración de los turnos de enfermería en las unidades de internamiento del Hospital Israelita Albert Einstein. Métodos: Fue desarrollado el Sistema de administración y Optimización de Recursos en la plataforma tecnológica dot.Net de Microsoft ${ }^{\circledR}$. Resultados: Fue implantado inicialmente en el área de enfermería con visualización on-line de los turnos de las unidades y respectivas demandas de pacientes. Facilita y permite la elaboración y administración de los turnos mensuales de trabajo, reduciendo el tiempo utilizado en la confección, garantizando de este modo seguridad en la información y agilidad en la obtención de datos. Conclusión: Después de la implantación es posible observar ganancias y oportunidades de mejoría tanto en el sistema como en el proceso de planificación y administración de los turnos de enfermería.

Descritores: Personal de enfermería en hospital; Sistemas de información; Admisión y programación de personal; Sistemas de información para admisión y escalafon de personal

\footnotetext{
* Trabalho realizado no Hospital Israelita Albert Einstein, São Paulo (SP), Brasil.

${ }^{1}$ Graduação em Enfermagem e Obstetrícia pela Escola de Enfermagem da Universidade de São Paulo, Brasil. MBA - Gestão em Saúde pelo IBMEC São Paulo (SP), Brasil.

${ }^{2}$ Graduação em Tecnologia de Processamento de Dados pela Faculdade de Tecnologia de São Paulo - FATEC, São Paulo (SP), Brasil.
} 


\section{INTRODUÇÃO}

Os avanços tecnológicos trouxeram modificações importantes nas mais diversas atividades desempenhadas pelo homem ${ }^{(1)}$. Na área da saúde não foi diferente. No Brasil, alguns hospitais começaram a instalar e a utilizar computadores no início da década de 80. Na enfermagem, as primeiras publicações foram na área da educação, por volta de $1985^{(2)}$. Desde então, o uso da tecnologia tem sido cada vez mais necessário nos hospitais e na enfermagem. Diversos sistemas estão sendo desenvolvidos, auxiliando os profissionais, tanto na assistência como na administração da assistência, no ensino e na pesquisa ${ }^{(2)}$.

$\mathrm{Na}$ administração da assistência, encontramos algumas iniciativas no Brasil relacionadas ao uso da informática para suporte na distribuição da equipe de enfermagem nas unidades de internação em hospitais ${ }^{(3-6)}$.

A enfermagem, dentro de um hospital, por atuar nas 24 horas e nos 7 dias da semana, trabalha em sistema de escalas. Assim a escala mensal, os dias de descanso (folgas e férias) de cada profissional da equipe devem ser planejados e distribuídos de forma racional, no sentido de garantir o número suficiente de cada categoria (enfermeiros e auxiliares/técnicos de enfermagem) para a assistência ao paciente. Sua elaboração deve ser sustentada pelas necessidades e características da clientela (perfil, demanda e padrões de sazonalidade), pelas características da equipe de enfermagem, dinâmica das unidades e legislação vigente. Além disso, reforça que esta elaboração é um processo complexo, e que exige tempo de quem a faz ${ }^{(7)}$.

A forma como as folgas são distribuídas nas escalas de enfermagem tem impacto direto na satisfação dos profissionais. A ausência de folgas nos finais de semana é fator de grande insatisfação ${ }^{(8-9)}$. Além disso, vários estudos mostram o impacto do número inadequado de profissionais de enfermagem nos resultados da assistência, como erros de medicação, quedas, infecções e mortes ${ }^{(10-14)}$.

A escala diária de enfermagem ou gerenciamento diário das escalas é realizada, antes da troca de cada turno, com o objetivo de garantir que os recursos humanos planejados e necessários estejam disponíveis para assistência ao paciente no próximo turno. Para tanto, é necessária uma análise quantitativa da relação do número de funcionários disponíveis (exclui-se das escalas planejadas os profissionais com ausências não previstas, como faltas, licenças, suspensões e outros) com o número de pacientes do período inclusive sua provável oscilação no plantão, e uma análise qualitativa que considera a carga de trabalho gerada pelos pacientes no turno.

Neste contexto, a liderança de enfermagem do Hospital Israelita Albert Einstein (HIAE) sentiu a necessidade de desenvolver um sistema eletrônico que pudesse auxiliar na confecção das escalas mensais de enfermagem e no gerenciamento diário destas otimizando os recursos humanos sem comprometer a qualidade da assistência de enfermagem.

Para fins deste estudo, planejamento, gerenciamento e otimização são processos relacionados à confecção e administração diária das escalas mensais de profissionais.

O objetivo deste artigo é relatar a desenvolvimento e implantação de um sistema informatizado para auxiliar no planejamento, gerenciamento e otimização das escalas de enfermagem nas unidades de internação do HIAE.

\section{MÉTODOS}

O HIAE é um hospital geral com 489 leitos divididos em unidades de clínica médico-cirúrgica (CMC), day clinic, oncologia, maternidade, berçário, pediatria, unidades de terapia intensiva e semi-intensiva adulto, pediátrico e neonatal.

Até fevereiro de 2005, a escala mensal dos profissionais de enfermagem era realizada nas unidades, pela liderança de enfermagem, em planilha Excel e disponibilizada em papel. Os ajustes diários necessários ou solicitados pelos profissionais, como trocas de horários e folgas, entre outros, eram realizados manualmente, pela liderança, nesta mesma planilha, resultando, ao final de cada mês, em escalas rasuradas e de difícil análise. Nesta metodologia de trabalho, a visualização da disponibilidade dos profissionais e da demanda de pacientes era local.

O Sistema de Gerenciamento e Otimização de Recursos (SGOR) foi desenvolvido no HIAE na plataforma tecnológica dot.Net da Microsoft ${ }^{\circledR}$, tendo o Visual Studio.Net como ferramenta, Asp.Net como linguagem e SQL Server 2000 como banco de dados. A decisão de desenvolver o sistema na instituição foi sustentada pela falta, no mercado, de produtos que atendessem às necessidades identificadas.

Para a construção do sistema foi formado um grupo de trabalho com profissionais das áreas de Enfermagem e de Tecnologia de Informação e definido um cronograma com cinco fases (planejamento, análise, desenvolvimento, homologação e entrega) com seis meses de prazo para finalização.

No planejamento foi realizada a identificação das necessidades. Dentre as necessidades destacadas estão a visualização on-line das escalas, o agrupamento destas por departamento, categoria profissional e turno de trabalho e a otimização da alocação dos profissionais nas áreas e departamentos.

$\mathrm{Na}$ fase de análise foi definida a construção do SGOR em dois módulos: o de Escala eletrônica, para substituir a escala mensal realizada em Excel e o de Gerenciamento, para realizar cálculos da otimização, entre eles a da relação paciente-enfermagem por categoria profissional, utilizando informações da escala mensal e dos demais sistemas institucionais. 
A opção de utilizar apenas a relação numérica baseou-se na ausência de um método de avaliação de carga de trabalho. Desta forma, a relação paciente-enfermagem foi estabelecida em conjunto com as lideranças das unidades de internação, por meio da análise do histórico da demanda e do tipo de pacientes internados por unidade e por turno de trabalho.

Assim, estabeleceu-se a relação paciente-enfermeiro e paciente-técnico/auxiliar de enfermagem considerada padrão por unidade e turno de trabalho. A Figura 1 mostra a relação do módulo de Gerenciamento com o módulo Escala Eletrônica e os demais sistemas da instituição.

O desenvolvimento do sistema foi realizado pelos profissionais da TI, com base nas informações das fases de planejamento e análise.

Para a homologação foi realizado um amplo plano de testes e validação de cada módulo do sistema desenvolvido. Por fim, o sistema foi oficialmente entregue e liberado para as áreas na medida em que os treinamentos foram realizados.

\section{RESULTADOS}

O SGOR foi implantado, inicialmente, para os profissionais de enfermagem em março de 2005 nas unidades da CMC e centro de terapia-intensiva adulto (semiintensiva e terapia intensiva). Entre agosto e dezembro de 2005 na pediatria (unidade de internação, semi-intensiva, terapia intensiva e ambulatório), berçários (normais, cuidados intermediários e terapia intensiva), maternidade, centro cirúrgico, oncologia (unidade de internação e ambulatórios de radio e quimioterapia) e unidade de primeiro atendimento e em janeiro de 2006 para fisioterapeutas, com abrangência neste período de 1.393 profissionais.

O SGOR foi disponibilizado na intranet do HIAE para todos os usuários. Para a segurança no manejo das informações, foram definidos perfis de acesso diferentes para os usuários, de acordo com cargo e função.

O módulo de Escala Eletrônica permite a realização das escalas mensais por área e por categoria profissional e a visualização on-line para todos os profissionais (Quadro 1). São utilizadas siglas que permitem o entendimento dos profissionais quanto aos dias referentes ao trabalho, remanejamento para outras unidades, folga, curso, treinamento, entre outros, com cores distintas para facilitar a visualização. Além disso, é possível diferenciar os profissionais que realizam as coberturas de férias e folgas pelos nomes nas cores azul e verde, respectivamente. $\mathrm{O}$ sistema registra o número de profissionais atuando no período por unidade (soma inclusive os que vêm de outros setores para auxiliar) e, no geral, por agrupamento definido pelo administrador (no caso da Quadro 1, CMC).

As escalas são criadas pelo sistema com base nas escala do mês anterior, isto é, o sistema organiza a escala de uma unidade com os mesmos profissionais. Cabe à liderança excluir ou incluir novos profissionais de acordo com seu planejamento.

A integração com o sistema institucional de cadastro de pessoal facilita a inclusão, na escala, do novo funcionário contratado e fornece acesso automático aos mesmos para que, por meio de senhas individuais, visualizem as escalas, solicitem folgas e troca de folgas. $\mathrm{O}$ sistema também recebe informações sobre as áreas em que o profissional está treinado e habilitado a trabalhar e quais áreas são restritas para este profissional.

Com a implantação do módulo Escala Eletrônica, o planejamento da escala mensal ocorre em três fases:

Fase 1 - Solicitação de folgas pelos funcionários

O sistema é liberado pelo administrador, para que os funcionários solicitem folgas. Para que se diferenciem as folgas

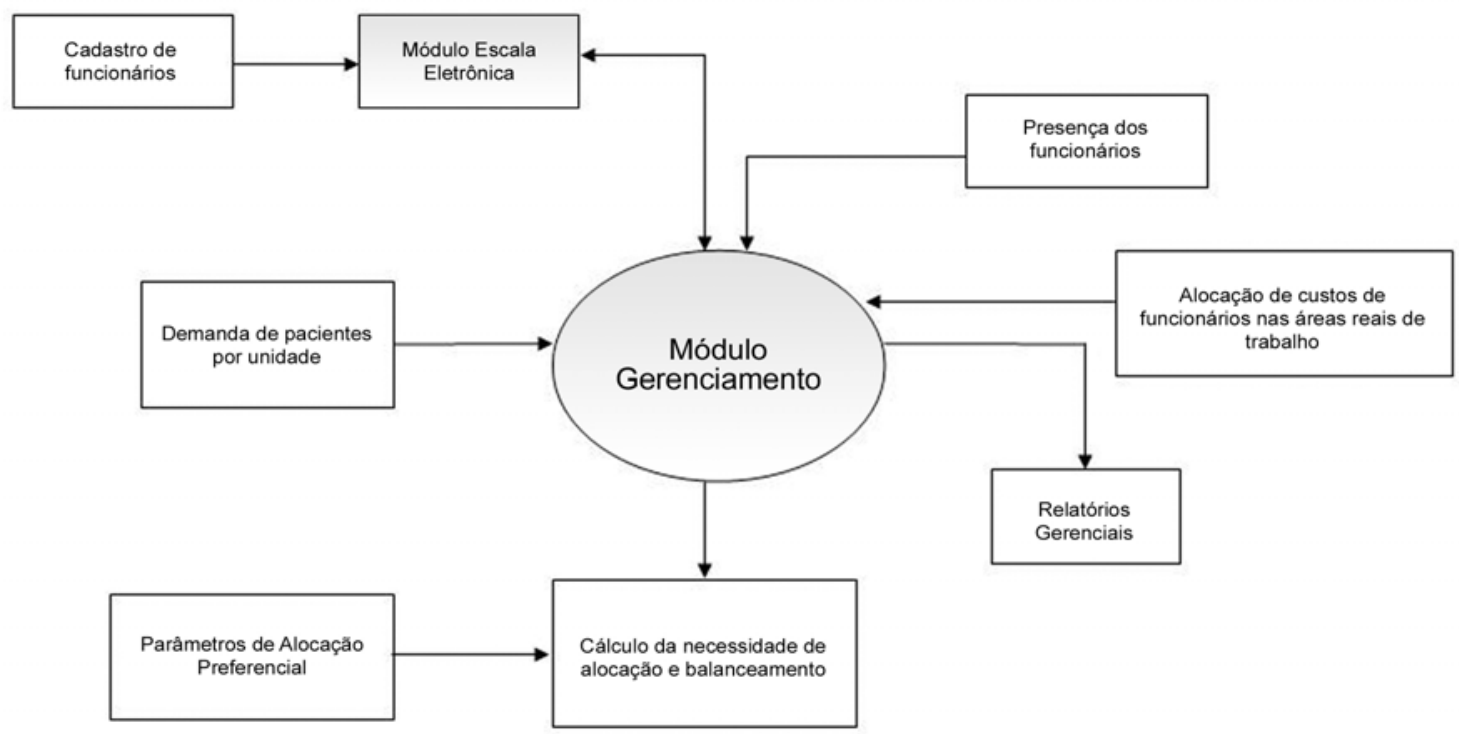

Figura 1 - Sistema de Gerenciamento e Otimização de Recursos (SGOR) 


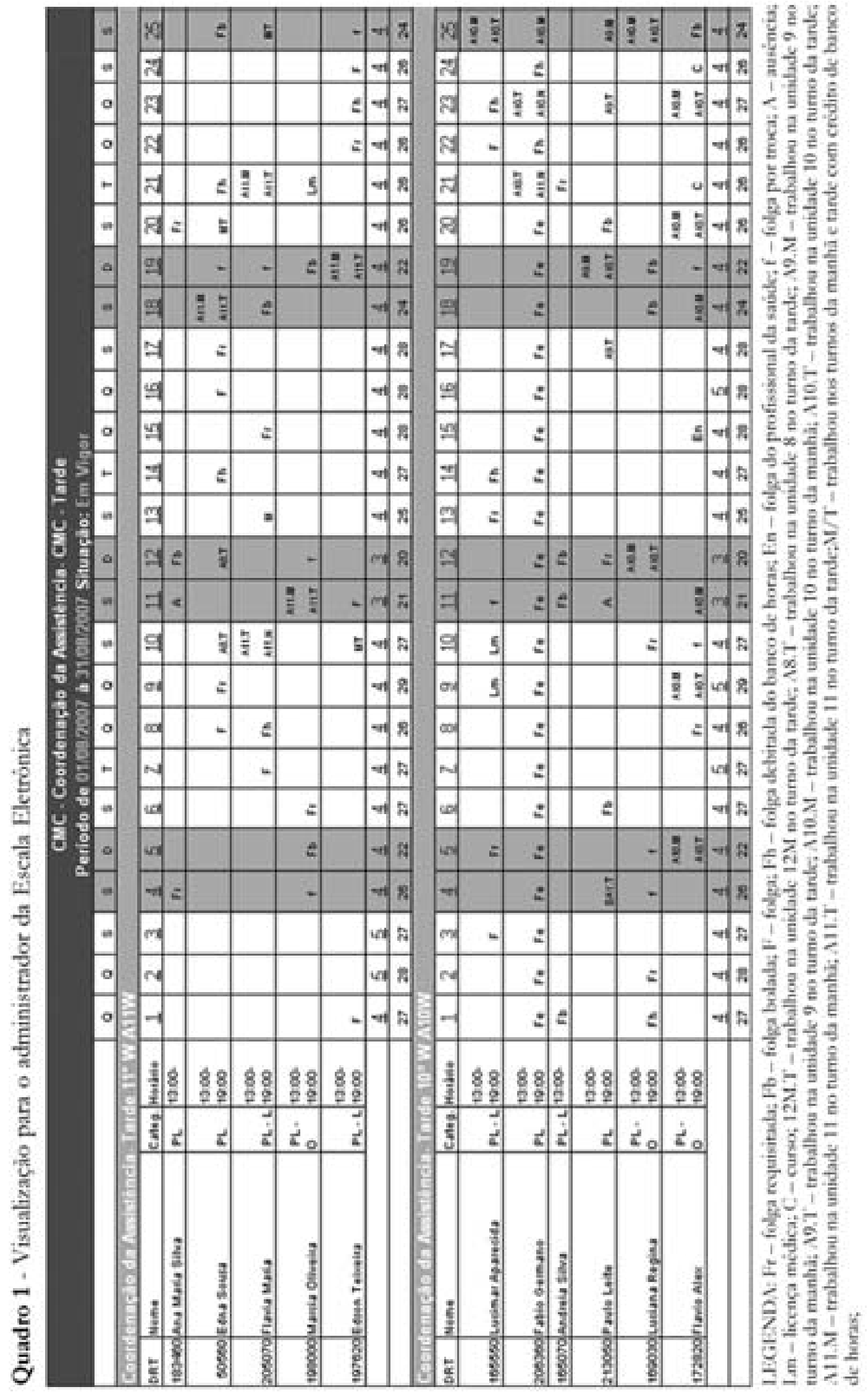


Quadro 2 - Módulo Gerenciamento: análise das escalas e sugestão de remanejamento

Análise por Departamento

\begin{tabular}{|c|c|c|c|c|c|c|}
\hline $\begin{array}{l}\text { Data para Cálculo: } \\
\text { Período: }\end{array}$ & $\begin{array}{l}2007 / 08 / 03 \\
\text { Tarde }\end{array}$ & & $\begin{array}{l}\text { Hora para C } \\
\text { Categoria : }\end{array}$ & $\begin{array}{l}13: 0 \\
\text { Enfe }\end{array}$ & eiros & \\
\hline Departamento & Área & $\begin{array}{c}\text { Total de } \\
\text { Pacientes Área }\end{array}$ & $\begin{array}{l}\text { Profissionais } \\
\text { disponiveis }\end{array}$ & $\begin{array}{c}\text { Mínimo de } \\
\text { Profissionais } \\
\text { necessários }\end{array}$ & $\begin{array}{c}\text { Máximo de } \\
\text { Profissionais } \\
\text { necessários }\end{array}$ & Resultado cálculo \\
\hline \multirow[t]{11}{*}{ CMC } & A05B & 15 & 3 & 2 & 2 & 1 \\
\hline & A05C & 0 & 2 & 0 & 0 & 2 \\
\hline & A08E & 15 & 2 & 1 & 1 & 1 \\
\hline & AOSW & 39 & 4 & 4 & 4 & $\mathrm{OK}$ \\
\hline & A10W & 41 & 4 & 4 & 4 & $\mathrm{OK}$ \\
\hline & A11E & 0 & 0 & 0 & 0 & $\mathrm{OK}$ \\
\hline & A11W & 14 & 5 & 2 & 2 & 3 \\
\hline & A12M & 8 & 1 & 2 & 2 & -1 \\
\hline & A7M & 14 & 2 & 1 & 1 & 1 \\
\hline & Day & 13 & 5 & 5 & 5 & $\mathrm{OK}$ \\
\hline & Geri & 15 & 2 & 2 & 2 & $\mathrm{OK}$ \\
\hline \multirow[t]{3}{*}{ Oncologia } & AOGW & 24 & 4 & 3 & 3 & 1 \\
\hline & QUIM & 0 & 4 & 4 & 4 & $\mathrm{OK}$ \\
\hline & RADIO & 0 & 3 & 2 & 2 & 1 \\
\hline
\end{tabular}

Sugestões de Indicação

\begin{tabular}{|c|c|c|c|c|c|c|}
\hline Departamento & Todos os Departamentc & & & Solicitar Indicação & & \\
\hline $\begin{array}{c}\text { Departamento } \\
\text { Deficitário }\end{array}$ & Área Deficitária & $\begin{array}{c}\text { Departamento } \\
\text { Cedente }\end{array}$ & Área Cedente & Profissional Selecionado & Prioridade & Realocar \\
\hline CMC & A12M & CMC & A05B & Jose Silva & 7 & Realocar \\
\hline$C \mathrm{C}$ & RA $5^{\circ}$ & $\mathrm{CC}$ & $\mathrm{CC} 2$ & Tiago Junqueira & 6 & Realocar \\
\hline Maternidade & M8 & Maternidade & M9 & João Oliveira & 2 & Realocar \\
\hline
\end{tabular}

consideradas prioritárias, o sistema permite que o profissional utilize siglas, que o administrador visualiza como Fr (folga requisitada) ou $\mathrm{Fb}$ (folga bolada) que sinaliza as consideradas essenciais (Figura 2). Ainda nesta fase, o funcionário pode adicionar um texto livre justificando suas solicitações.

\section{Fase 2 - Confecção das escalas}

Nesta fase, o sistema é bloqueado para o funcionário e o administrador realiza as escalas, sinalizando as folgas alteradas ou inseridas, os cursos, os treinamentos e outras situações como alteração do horário e de setor, com siglas que facilitem o entendimento do funcionário.

O sistema realiza, ainda, uma análise das escalas em relação ao número de folgas permitidas e número de folgas nos finais de semana, parametrizados pelo administrador de acordo com legislação vigente ou normas da instituição.

Ao finalizar esta fase, a escala é considerada vigente e liberada para a visualização dos funcionários.

\section{Fase 3 - Escala em vigor}

Ao longo desta fase, os funcionários podem solicitar trocas dos dias definidos como folgas. $\mathrm{O}$ administrador do sistema pode aceitar ou recusar esta solicitação. Em caso positivo, o sistema faz a alteração, automaticamente, na Escala Eletrônica.

O módulo de Gerenciamento realiza o controle diário das escalas planejadas, calculando a relação real de pacienteenfermeiro e paciente-técnico/auxiliar de enfermagem disponível para a assistência, indicando as unidades em que há sobra e falta de profissionais, de acordo com a relação padrão estabelecida, para a realocação ou não pelo administrador (Quadro 2).

Para tanto, o módulo de Gerenciamento interage com o módulo da Escala Eletrônica, que indica o número de funcionários disponíveis, e com o sistema eletrônico institucional, que o alimenta, com o número de pacientes internados (Figura 1).

A implantação do SGOR também resultou no armazenamento eletrônico dos dados, permitindo, de forma segura e rápida, a mensuração, a análise e a formatação de série histórica, possível anteriormente com utilização de muitos recursos e tempo.

Ainda, esta tecnologia trouxe a visualização on-line, tanto das escalas das unidades quanto das respectivas demandas de pacientes, possibilitando o remanejamento dos profissionais entre as unidades, o que permitiu uma relação uniforme de profissionais por pacientes e a transparência de todo o processo.

\section{DISCUSSÃO}

A utilização de tecnologia no planejamento e 
gerenciamento dos profissionais de enfermagem no HIAE trouxe a necessidade de revisão dos processos e de mudança na cultura relacionados a estas tarefas. $\mathrm{O}$ aumento no uso da tecnologia computacional vem apresentando um impacto na profissão de enfermagem o que influencia como estes profissionais desenvolvem seu trabalho ${ }^{(15)}$ sendo necessário que novos papeis sejam desempenhados ${ }^{(3)}$.

Acredita-se que a utilização do SGOR em 100\% da equipe de enfermagem das unidades de internação esteja relacionada a diversos fatores também descritos na literatura, entre eles o envolvimento das lideranças no processo de desenvolvimento do sistema ${ }^{(3)}$, a redução no tempo utilizado na confecção das escalas ${ }^{(2-6)}$, a possibilidade do acesso às escalas por qualquer computador na instituição e a visualização do número de profissionais na assistência ${ }^{(6)}$.

Assim como Aurich ${ }^{(4)}$, os autores do presente estudo entendem que a possibilidade da geração de base de dados sólida que permita a obtenção de relatórios e informações automáticas, pode contribuir para pesquisas e análises relacionadas a dimensionamento, absenteísmo, horas trabalhadas e horas extras. Outras facilidades observadas após a implantação do SGOR foram a possibilidade de visualização da demanda de pacientes para cálculo da relação enfermagem-paciente e indicação de remanejamento entre unidades, que não estão descritas na literatura consultada.

\section{REFERÊNCIAS}

1. Druker P. Além da revolução da informação. HSM Management. 2000;3(18):48-55.

2. Marin HF. Informática em enfermagem. São Paulo: EPU; 1995.

3. Sottomaior VS, Ferreira LAA, Funcke LB. Desenvolvimento e implantação do sistema informatizado para a escala mensal do pessoal de enfermagem do Hospital das Clínicas de Porto Alegre. Rev HCPA \& Fac Med Univ Fed Rio Gd do Sul. 1998;18(2):197-203.

4. Aurich EA. Informatização de tarefa administrativo-burocrática em enfermagem: proposta de construção de protótipo de sistema de elaboração de escala mensal de pessoal [dissertação]. São Paulo: Escola de Enfermagem da Universidade de São Paulo; 2002.

5. Rangel AL. Desenvolvimento de um sistema de apoio à decisão para a elaboração da escala periódica de pessoal de enfermagem [dissertação]. Ribeirão Preto: Escola de Enfermagem de Ribeirão Preto da Universidade de São Paulo; 2006.

6. Magalhães ZR, Gonçalves L, Faria GF, Dahi AC, Nascimento ECS. Disponibilização em rede da escala de trabalho da equipe de enfermagem do Hospital das Clínicas da UFMG. In: $8^{\circ}$ Simpósio Brasileiro de Comunicação em Enfermagem, São Paulo, Maio 2002 [Internet]. [citado 2007 Ago 9]. Disponível em: http:/ / www.proceedings.scielo.br/ pdf/sibracen/n8v1/v1a063.pdf

7. Massarollo MCKB. Escala de distribuição de pessoal de enfermagem. In: Kurcgant P, coordenadora. Administração em enfermagem. São Paulo: EPU; 1991. p.107-15.

8. Ruggiero JS. Health, work variables, and job satisfaction among nurses. J Nurs Adm. 2005;35(5):254-63.
Adicionalmente, observou-se o interesse de outras categorias, como fisioterapeutas e nutricionistas, em utilizar o sistema da instituição. Autores ${ }^{(3)}$, também descrevem a utilização do sistema informatizado de escala mensal do pessoal de enfermagem desenvolvido em seu hospital para outros setores da instituição.

O próximo passo é realizar melhorias no sistema, principalmente na interação com o ponto eletrônico dos funcionários e com um sistema que calcule a carga de trabalho gerada pelos pacientes.

\section{CONCLUSÃO}

Após dois anos da implantação do SGOR, é possível observar ganhos e oportunidades de melhoria, tanto no sistema como no processo de planejamento e gerenciamento das escalas de enfermagem.

\section{CONSIDERAÇÕES FINAIS}

O SGOR não deve ser considerado apenas como uma ferramenta para otimizar as atividades administrativas dos enfermeiros. Cabe às lideranças de enfermagem analisar os dados gerados por este sistema e transformálos em informação, para o auxílio na argumentação e tomada de decisão, como uma das estratégias que corroboram com a segurança do paciente.

9. Marquis BL, Huston CJ. Leadership roles and management functions in nursing: theory and application. 3rd ed. Philadelphia: Lippincott; 2000. Staffing needs and scheduling policies; p. 262-77.

10. Joint Commission Resources. Providing an adequate number and skill mix of staff. In: Joint Comission Resources. Issues in human resourses for hospitals. 2004. p. 57-81.

11. Lankshear AJ, Sheldon TA Maynard A. Nurse staffing and healthcare outcomes: a systematic review of the international research evidence. ANS Adv Nurs Sci. 2005;28(2):163-74.

12. McCue B, Mark BA, Harless DW. Nurse staffing, quality, and financial performance. J Health Care Finance. 2003;29(4):54-76.

13. Aiken LH, Clarke SP, Sloane DM, Sochalski J, Silber JH. Hospital nurse staffing and patient mortality, nurse burnout, and job dissatisfaction. JAMA. 2002; 288(16):1987-93. Comment in: Am J Nurs. 2003;103(1):22. JAMA. 2002;288(16):2040-1. JAMA. 2003;289(5):549-50; author reply 550-1. JAMA. 2003;289(5):549; author reply 550-1. JAMA. 2003;289(5):550; author reply 550-1.

14. Needleman J, Buerhaus P, Mattke S, Stewart M, Zelevinsky $K$. Nurse-staffing levels and the quality of care in hospitals. N Engl J Med. 2002;346(22):1715-22. Comment in: N Engl J Med. 2002;346(22):1757-66. N Engl J Med. 2002;347(14):1118-9. N Engl J Med. 2002;347(14):1118-9. N Engl J Med. 2002;347(14):1118-9. N Engl J Med. 2002;347(14):1118-9.

15. Évora YDM, Fávero N, Trevizan MA, Melo MRAC. Evolução histórica da aplicação do computador na enfermagem (1965-1998). Acta Paul Enf. 2000; 13(N Esp Pt 2):143-7. 